\title{
Current looping NEXRAD is inadequate for hazardous-weather flight
}

\author{
William R. Knecht \\ Civil Aerospace Medical Institute, \\ Federal Aviation Administration \\ Oklahoma City, OK, USA \\ william.knecht@faa.gov
}

\begin{abstract}
Next-Generation Radar (NEXRAD) is an important decision-support tool for air traffic control (ATC). It is currently being introduced into aircraft cockpits, and will be equally important for remote piloting of unmanned aerial systems (UAS). Unfortunately, numerous studies strongly suggest that manually estimation of closest point of approach (CPA) to heavy weather is a difficult task for pilots, and that current-generation NEXRAD may be intrinsically inadequate for that task. In this study, we begin with an ecological information analysis of the looping NEXRAD format itself, and show that inherent task difficulty is predicted mathematically and by the neural structure of the visual system. Then, to confirm empirically, we develop a mathematical model of an extremely simple "ideal storm" to generate various types of simulated weather cells. A looping NEXRAD-type parttask simulation is created, and 21 general aviation (GA) pilots are tested to measure effects of weather system depth (19 vs. $40 \mathrm{~nm})$, and the opening and closing of gaps at various closure rates between weather cells $(-14,-7,0,7$, $14 \mathrm{kt})$. For the values tested, weather system depth has no significant effect on eventual CPA from heavy weather ( $>40 \mathrm{dBZ}$ reflectivity). However, redesign and retesting may be warranted for this variable. In contrast, weather movement clearly and greatly degrades safety. It does not seem to matter if weather movement is as slow as 7 scale kt, nor whether gaps are opening or closing. Any weather movement makes an already hard task harder. We can now say with certainty that current-generation looping NEXRAD is inadequate to manually keep aircraft safely separated from weather. Ecological analysis indicates necessity of adding 30-45 minutes of future-predicted (forecast) weather and a $20 \mathrm{~nm}$ range ring to the looping NEXRAD display to enable safe tactical weather avoidance.
\end{abstract}

Keywords—aviation; weather; NEXRAD; safety; air traffic control; avionics; UAS; ecological interface design; direct perception; human factors

\section{INTRODUCTION}

Adverse weather remains a perennial challenge for aviation and a high priority for the U.S. Federal Aviation Administration (FAA). The FAA Next Generation (NextGen) Air Transportation System Implementation Plan discusses "up-to-date weather and airspace status information delivered directly to the cockpit" as part of its mission [1]. One important focus area involves pilot interpretation and use of color-coded weather-risk displays. In the U.S., the best-known of these is the National Weather Service (NWS) NEXRAD.

GA pilots and UAS operators are now being offered NEXRAD capability, for instance via incockpit XM satellite radio, and on handheld devices like tablet computers and smartphones. From a human-factors perspective, NEXRAD contains important perceptual information that pilots can use to make hazard-avoidance decisions $[2,3]$ - particularly, how close their flightplan may take them to hazardous weather.

Normally, NEXRAD images are updated about once every five minutes. Rapid playback of about an hour's worth of 5-minute-interval data is enough to create a time-lapse movie conveying apparent motion [4] indicating where a storm is heading.

Nevertheless, looping NEXRAD ultimately shows a movie of where precipitation used to be. At issue is whether that information can be used to predict where both the aircraft and hazardous weather will be in the near future.

We know that pilots can estimate closest point of approach to storms on NEXRAD. But, in every study to date (all in simulo), a substantial proportion seemed to overestimate CPA, meaning they overestimated eventual minimum separation from heavy weather, and ended up approaching too closely $(2,5,6,7,8,9,10,11,12,13,14]$. In no study did all pilots consistently maintain the $20 \mathrm{~nm}$ separation from heavy weather advised in FAA Advisory Circular AC 00-24-C [15].

To investigate why, we first took a theory-based look at the visual information present in looping NEXRAD. Afterward, we tested pilots in near- 
ideal conditions, to get an idea of what bestpossible performance might look like.

\section{A. Ecological information analysis}

Some perceptual tasks are so easy that almost no training is required to produce high performance. Vernier acuity is an example [16]. Yet, such is not the case with looping NEXRAD.

Ecological psychology [17] encourages us to examine what information in a visual stimulus might enable a given task. For example, what in a NEXRAD display might allow us to keep an aircraft icon 20 scale miles away from a storm? Once we know that critical information, neurocomputation [18] prompts us to consider whether this information could be either directly detected and/or realistically "computed" by structures of neurons.

Avoiding a single point on a storm's edge is the simplest possible case of "weather avoidance." In reality, there would be many such points to consider, but we can consider just one because their logic will be similar.

Given the simplest case of straight-line, constant-velocity movement, the parametric equation describing the range $r_{t}$ between the aircraft icon and a single, moving point on the "ideal" storm at time $t$ is

$$
r_{t}=\sqrt{\left(x_{0}+v_{x} t\right)^{2}+\left(y_{0}+v_{y} t\right)^{2}}
$$

$x_{0}$ and $y_{0}$ being initial relative separation distances (e.g., $x_{0}=x_{0 \text { aircraft }}-x_{0 \text { storm }}$ ), and $v_{x}$ and $v_{y}$ the relative-velocity components (e.g., $v_{x}=v_{x \text { aircraft }}$ - $v_{x \text { storm }}$ ), all of which can be derived by comparing at least two views of the situation, separated by a known amount of time.

Solving (1) for slope $d_{r} / d_{t}=0$ gives us CPA - the task-relevant information we need:

$$
C P A=\sqrt{\left(x_{0}^{2}+y_{0}^{2}\right)-\frac{\left(x_{0} v_{x}+y_{0} v_{y}\right)^{2}}{\left(v_{x}^{2}+v_{y}^{2}\right)}}
$$

This shows that - at least in the absolute simplest case-looping NEXRAD does theoretically contain sufficient information for pilots to know how close they will approach a storm boundary.

The problem comes in trying to imagine noisy neurons computing (2) to at least several decimal places. Neither does there seem to be any reasona- ble "hack," or shortcut way to estimate CPA (such as the pilot's old trick of knowing that they will eventually collide with a distant aircraft if it grows larger with time, but does not change relative position out the window). If we plot (1) over time, we see that all combinations of approach produce a Vshaped function showing almost no information about the second derivative (deceleration of the slope) until the aircraft is unsafely close to the hazard. This gives us no hack to estimate CPA. We could call this the rounded-V problem.

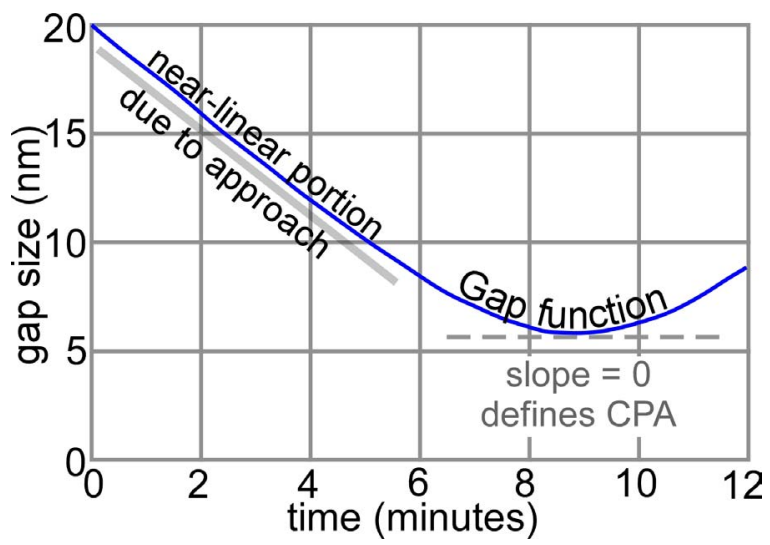

Fig. 1. Plot of (1), showing the rounded-V problem.

Finally, we also suspect that it will be easier to estimate CPA in static weather than in moving weather, if only because the relative velocities $x_{0}$ and $y_{0}$ should be easier to estimate.

\section{B. Empirical follow-up}

We first developed a method of generating a near-ideal, high-resolution, zero-data latency, extremely simple graphical heavy convective weather system and displaying it at a frame rate sufficiently high to convey good apparent motion. Then, if pilots were still unable to safely navigate past such weather, that would verify both a lack of effortless, accurate direct perception and a need to ecologically re-engineer NEXRAD displays to make them fit for storm avoidance.

\section{METHOD}

\section{A. Hardware}

Hardware consisted of laptop computers with an optical mouse and a 12 " $(30.5 \mathrm{~cm}) \times 7.5$ " $(19$ $\mathrm{cm}), 1280 \times 800$ pixel screen. Pilots self-seated at a comfortable distance.

Mathematica [19] was used to create and display NEXRAD-like movies consisting of a 
series of semi-transparent still images (frames) with synthetic weather information overlaid on a map of terrain and roads (Fig. 2). Mathematica also provided event handling (e.g., reading onscreen mouse clicks), to let participants draw their flightpath on-screen, capture the results, compute minimum CPA to heavy weather [20], and store the results to files readable by statistical software.

Full methodology is described in [8]. Briefly, this required independent control of at least two weather cells' a) initial location, b) size, c) shape, d) angle of orientation, e) movement, f) color scheme, and g) time-evolution. Importantly, weather cells had to maintain contiguity - the same order of colors - even while morphing across the screen. Red always had to sit next to orange, orange next to yellow, and so forth. The color scheme was made to resemble key color selections from the NWS WSR-88D Intensity Legend [21]. Note that orange represents $45 \mathrm{dBZ}$ reflectivity, and is actually the "heavy" weather FAA AC 0024-C advises pilots to avoid by at least $20 \mathrm{~nm}$.

\section{B. Software}

Three separate computer programs were written, a) Weather Generator, b) Viewer, and c) Autoscorer. The Weather Generator allowed control over shape, position, and movement of the two weather cells generated per frame, 12 frames per scenario, representing one hour's worth of weather history at 5-minute intervals. For the current work the lower-left cell was kept static, while the upper-right cell was constrained to lie on a diagonal, either a) directly approaching the lower cell, b) static, or c) moving directly away from it.

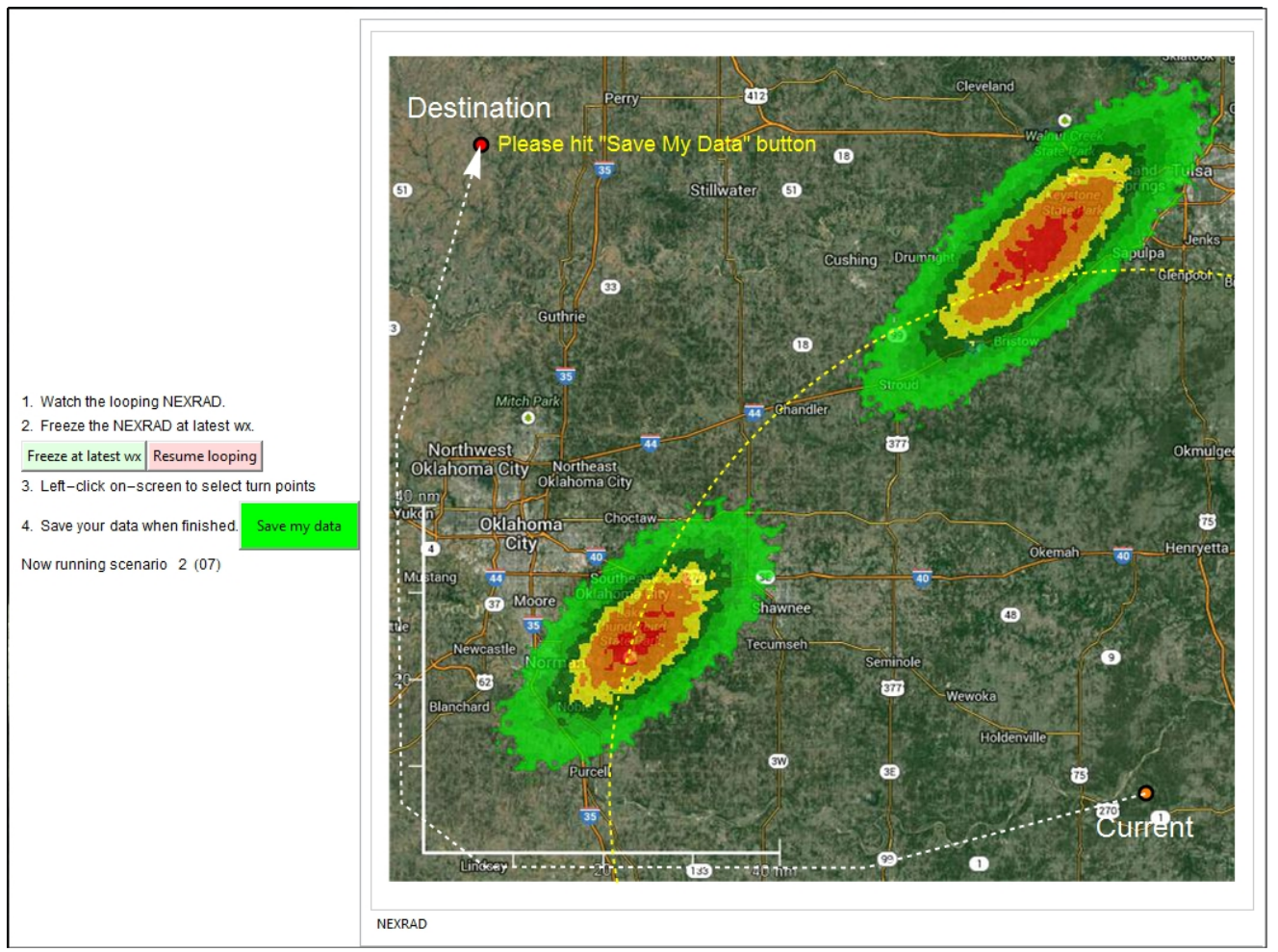

Fig. 2. Viewer, showing "shallow" (19 km deep) weather cells, and a sample flightplan with four inflection points. "Deep" cells were $40 \mathrm{~nm}$, and looked more circular.

The Viewer program (Fig. 2) displayed looping NEXRAD by sequencing each scenario's 12 images into a movie displayed at 2.2 frames per second (fps), close to the optimal rate of $3.0 \mathrm{fps}$ tested by Lemos and Chamberlain [10]. It displayed a scale of miles at lower left, a "Freeze" button, which stopped the movie at the most- current point in time, and a "Resume" button, to allow looping to resume. A dashed, yellow, circular dashed range ring depicted where the aircraft was expected to be in $1 / 2$ hour, given a stated, fixed airspeed of 120 ktPilots were instructed to create a constant-speed flightplan intended to maintain at least $20 \mathrm{~nm}$ clearance from 
"orange" weather. Each flightplan automatically began at the map point labelled "Current," representing the aircraft's current position at the most recent weather frame. To draw the flightplan, a pilot simply had to move the mouse cursor to a desired inflection point - a map location where they intended to turn-and left-click. The computer automatically drew a straight, dashed line from each inflection point to the next, and recorded the point's $x, y$ coordinates. Fig. 2 shows a sample flightplan.

The display was set up with no data latencyno time delay before the information reached the cockpit, such as exists in current NEXRAD [22]. The challenge was, therefore, to watch the evolution of weather up to the current time, then try to predict where both the aircraft and weather would be in the future, and plan a flight maintaining $\geq 20 \mathrm{~nm}$ clearance from where "orange" weather was estimated to be.

Finally, the Autoscorer program used numerical methods to computer-score the data for futureprojected CPA.

\section{Experimental design}

Two weather depths and five gap-opening speeds were set up in a $2 \times 5$ repeated-measures design with randomized scenario presentation order.

As stated earlier, a simplified "weather system" with only two weather cells was used. The lowerleft cell never moved, and was located to guarantee at least one safe option for flying around the weather. The upper-right cell could only move diagonally, either toward or away from the lowerleft cell. Negative weather speeds therefore represented gaps closing over time, zero meant a static gap, and positive speeds meant opening gaps, all corresponding to simulated weather-movement speeds of $-14,-7,0,7$, or $14 \mathrm{kt}$. In the descriptions that follow, these speeds are labelled respectively as gap "closing-fast," "closing-slow," "static," "opening-slow," and "opening-fast."

There were two experimental hypotheses. First, if gaps could be slipped through quicker, risk exposure might be judged as lower. Hence, "shallow" (19 nm-depth) storms might reasonably tempt more gap-shooting than "deep" (40 nm) storms.
Second, gap-change speed might modulate perception of future gap size. Gaps increasing in size might tempt the most gap-shooting, static gaps, the next-most, with gaps decreasing in size, the least. To properly control for this confound, it was critically important to position the weather cells to equilibrate the size of gaps at the expected arrival time of the aircraft.

To that end, the speeds of the aircraft and weather were held constant, and the initial location of each scenario's moving weather cell was carefully placed to match both its speed and the aircraft's to always produce 20.1-20.5 nm safe clearance from "orange" weather at the time the aircraft would arrive should the pilot choose to "fly direct." However, none of the pilots were told this. They were only told that it was acceptable to go direct when it was safe.

\section{Participants}

GA pilots were recruited from a local Oklahoma City, OK area flight school. Table 1 shows certificates, ratings, age, and total flight hours (TFH) of the 21 pilots.

TABLE I. PILOT DEMOGRAPHICS ( $\mathrm{N}=21)$.

\begin{tabular}{|l|r|l|r|r|r|r|c|}
\hline \multicolumn{4}{|c|}{ Certificates and Ratings } & \multicolumn{4}{|c|}{ Age and Total Flight Hours } \\
\hline \multicolumn{1}{|c|}{ Cert/Rating } & \multicolumn{1}{|c|}{ Cert/Rating } & \multicolumn{1}{c|}{$\boldsymbol{N}$} & & Mean & Mdn & \multicolumn{1}{c|}{$\boldsymbol{D} \boldsymbol{D}$} \\
\hline Student & 8 & CFII & 2 & Age & 22.7 & 21.5 & 4.0 \\
\hline Private pilot & 13 & Commercial & 2 & & & & \\
\hline Instrument-rated & 4 & Multi-engine & 2 & $\boldsymbol{T F H}$ & 176.5 & 137.4 & 185.4 \\
\hline CFI & 3 & ATP & 0 & & & & \\
\hline
\end{tabular}

\section{RESULTS}

Fig. 3 summarizes the 21 pilots' CPAs for the 10 scenarios. Pilots performed best in the staticweather condition. Weather depth was nonsignificant.

Repeated-measures analysis of variance on all 10 scenarios (Fig. 3) nearly failed $\left(p_{M}=.058\right)$ Mauchly's test of sphericity (a test of the appropriateness of using analysis of variance), and glaringly so for the interaction depth $\times$ speed $\left(p_{M}=.001\right)$. Given the nearly circular shape of our "deep" weather cells, redesign of those may be warranted. 


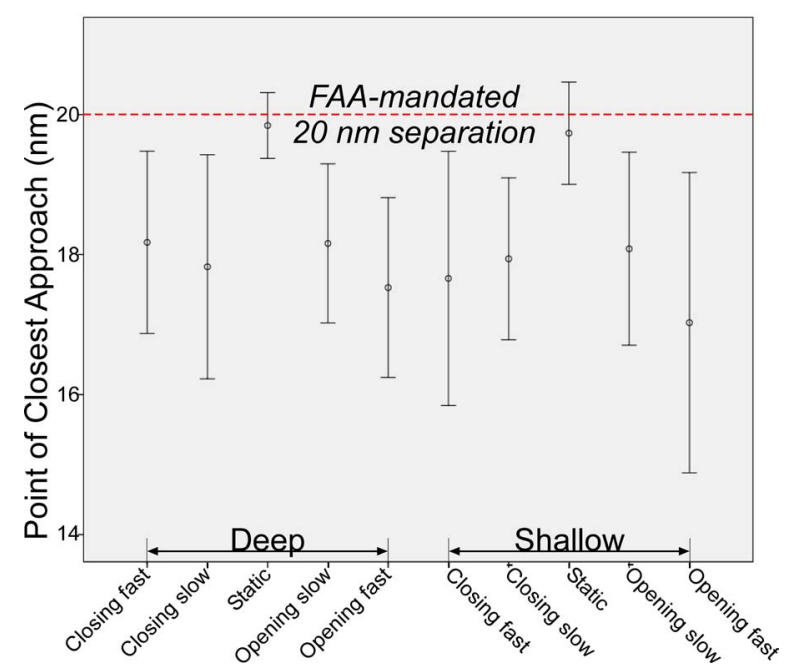

Fig. 3. CPA to "orange" weather for "deep" vs "shallow" weather cells, and five gap-closure speeds. Error bars show normality-assumed 95\% confidence intervals.

We therefore dismissed width and the interaction, collapsed (averaged) the data across width, and reanalyzed speed alone with the nonparametric Friedman test. This showed rankordered CPAs differing significantly on gap speed $\left(p_{\text {Friedman }}=.016\right)$. Fig. 4 shows the boxplots.

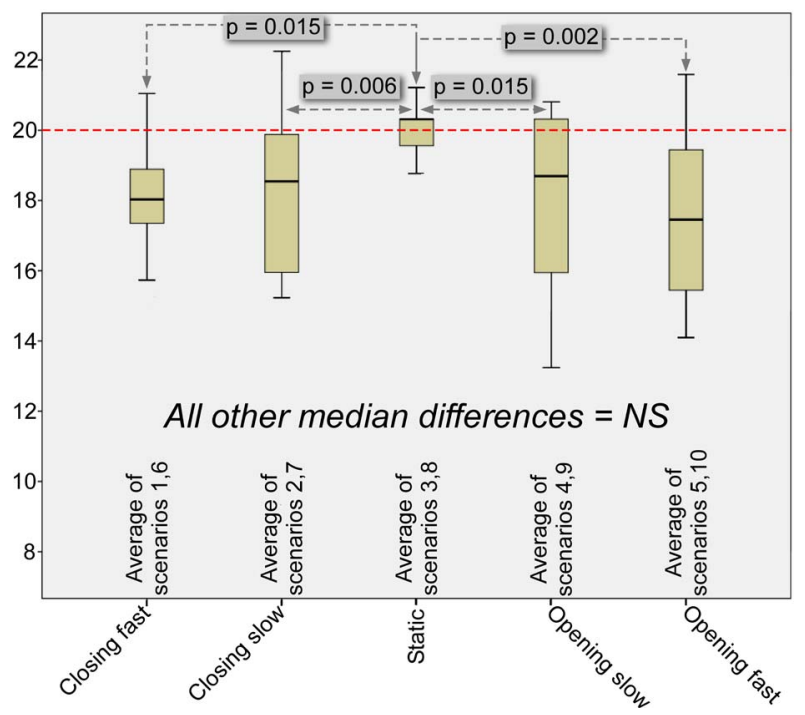

Fig. 4. Boxplots of gap-closure speed collapsed across weather-cell depth. Error bars show actual 95\% intervals.

An alternate way to examine these data is to count pilots who "flew direct," shooting the (technically safe) gap between weather cells. Here, a pattern of hits emerged similar to Fig. 3. Therefore, results were again collapsed across width.

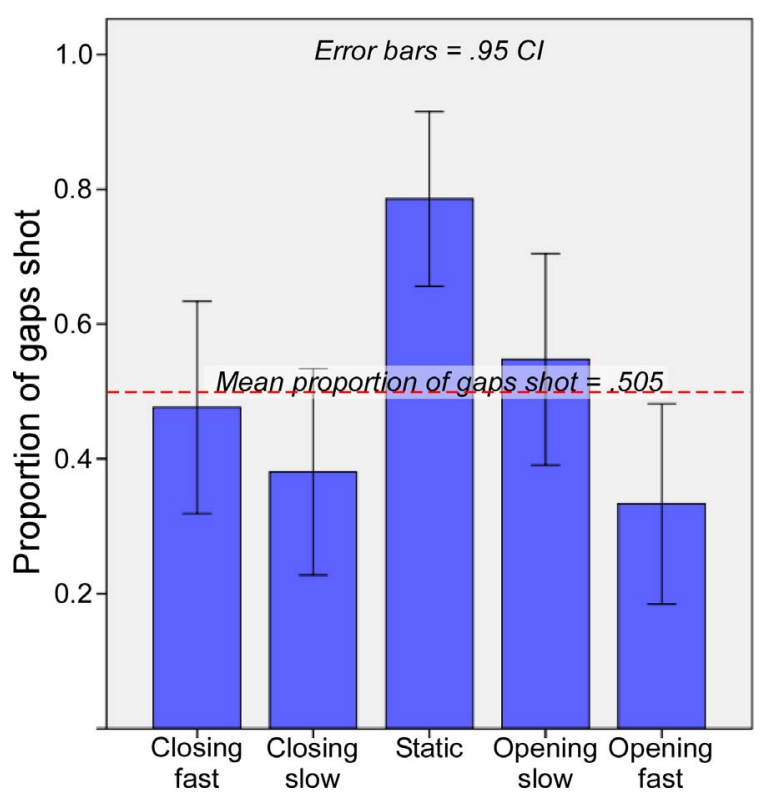

Fig. 5. Proportions of gaps shot.

Fig. 5 shows how the greatest number of gaps shot-again-occurred when both weather cells were static $\left(p_{X}^{2}=.033\right)$.

\section{DISCUSSION}

NEXRAD is now finding its way into GA aircraft cockpits. However, strong evidence from numerous studies suggests that many pilots using NEXRAD-like technology will approach too closely to convective weather. Even advanced predictive displays graphically showing short-term forecast weather, while helpful, still show a significant fraction of pilot participants not consistently maintaining FAA-mandated $20 \mathrm{~nm}$ clearance from heavy weather.

Looping NEXRAD seems a necessary improvement over single-frame display. But, whether it was sufficiently safe remained an open issue.

To investigate, we began with ecological information analysis, examining the information available in the visual stimulus. This revealed that Gibsonian direct perception of future closest point of approach (CPA) to hazard appears to be unlikely at on-screen ranges-to-hazard beyond a few scale miles, except in the case of very large, very stable storm systems where a CPA of zero is obvious and inevitable.

To empirically support the prediction that using looping NEXRAD should be difficult and inaccurate, we tested CPA estimation accuracy in 
the simplest possible case-a part-task display having no data latency and very simple, predictable weather. Mathematically generated convectiveweather radar images allowed precise control over the shape, placement, and movement of weather cells. This allowed testing two potentially important perceptual independent variablesweather system depth, and the opening and closing of gaps between cells.

Results indicated that judgment of CPA to heavy weather with this idealized looping NEXRAD did not differ significantly when comparing our shallow (19 $\mathrm{nm}$ deep) vs. deeper $(40 \mathrm{~nm})$ weather systems. However, given the limited, elliptical shape of our weather cells, further testing of weather depth is advised.

In contrast, weather movement clearly and significantly degraded safety. Predictably, CPA was greatest/safest in the static conditions where neither weather cell moved. In contrast, when even a single cell moved as slowly as $7 \mathrm{kt}$, no matter whether the gap between cells was opening or closing, judgment of future CPA deteriorated significantly.

As predicted by the ecological information analysis, the mere task of judging future CPA was extremely difficult no matter what the conditions. Table 2 summarizes pilot behaviors and outcomes.

Of the 210 total scenarios, only 85 (40.5\%) were successes, meaning that pilots maintained at least $20 \mathrm{~nm}$ clearance from heavy weather $(>40$ $\mathrm{dBZ}$ reflectivity). The remaining 125 (59.5\%) were failures.

Even though gaps were engineered to always be safe by the time the aircraft arrived, in 104 of 210 cases, pilots chose to divert around the entire storm system. Three of those 104 diversions were around the moving cell, all three resulting in catastrophic separation loss $(\mathrm{CPA}=0.004-10.104 \mathrm{~nm})$. Worse still, of the remaining 101 diversions around the static cell, 80 (79.2\%) still had CPAs less than 20 nm.

TABLE II. SUMMARY OF OUTCOMES

\begin{tabular}{|c|c|c|c|c|c|c|c|}
\hline & \multicolumn{3}{|c|}{ Successes } & \multicolumn{3}{|c|}{ Failures } & Total \\
\hline Pilot behavior & $n$ & Mean CPA (nm) & $S D(n m)$ & $n$ & Mean $C P A(n m)$ & $S D(n m)$ & \\
\hline Shot the gap between weather cell & 64 & 20.32 & 0.25 & 42 & 17.29 & 1.95 & 106 \\
\hline Diverted around static weather cell & 21 & 21.00 & 0.84 & 80 & 16.72 & 2.47 & 101 \\
\hline Diverted around moving weather cell & 0 & & & 3 & 4.09 & 5.32 & 3 \\
\hline Total & 85 & & & & & & 210 \\
\hline
\end{tabular}

In other words, even when simply plotting a course around a static storm cell, $79 \%$ of pilots failed to maintain proper clearance.

Training typically improves task performance. Obviously, it is necessary. But, would it ever be sufficient? To that we can now reply conclusively that the information presented by looping NEXRAD simply does not appear to adequately support the task, except in extremely limited circumstances. Moreover, the realworld task of safe navigation will only be much harder than ideal, given changing, chaotic storm systems moving faster than the ones tested here, not to mention the effect of 15-minute radar data latencies [9].

Addition of a range ring to the display would greatly alter the task, changing it from one of estimating gap size from the aircraft icon to CPA, to one of estimating gap size from the edge of the range ring to CPA. This would help eliminate the "rounded-V problem" described earlier (Fig. 1).

\section{CONCLUSION}

If the information in NEXRAD is insufficient, what will it take to enable safe navigation around heavy precipitation? The following summarizes what we strongly recommend on the basis of the best available knowledge.

1. Autopilot (to maintain accurate course and predictable speed)

2. Zoom and pan capabilities on the display

3. At least 30-minute historical/445-minute futureforecast capability from the NEXRAD data feed

4. Looping and/or scroll capability for \#3, with method of showing direct age of each frame 
5. Ownship icon, with a 20-nm range ring, that displays aircraft position from time $=$ now to $+\geq 30$ minutes

6. A route-planning feature showing estimated CPA, to test potential flightpaths.

7. Ultimately, an integrated hazard display to show convective weather, turbulence, icing, etc.

8. A full-system training curriculum

\section{ACKNOWLEDGMENT}

This study is supported through the FAA NextGen Human Factors Division, ANG-C1.

\section{REFERENCES}

[1] Federal Aviation Administration. (2012). "NextGen implementation plan," (p. 8), March, 2012.

[2] W.R. Knecht, and E. Frazier, "Pilots' risk perception and risk tolerance using graphical risk-proxy gradients," (Technical Report no. DOT/FAA/AM-15/9). Washington, DC: Federal Aviation Administration, 2015.

[3] M.W. Wiggins, D. Azar, and T. Loveday, "The relationship between pre-flight decision-making and cue utilization," Proc. Human Factors \& Ergonomics Soc., 56(1), pp. 2417-2421, 2012.

[4] M. Wertheimer, "Experimentelle studien über das sehen von bewegung,". Zeitschrift für Psychologie, 61, pp. 161-265, 1912.

[5] Atmospheric Technology Services Company, "Demonstration comparing the effects of probabilistic and deterministic forecast guidance on pilot decision-making and performance," (Report no. FAA-20130902.1). Washington, DC: Federal Aviation Administration., 2013.

[6] D.B. Beringer, and J.D. Ball, "The effects of NEXRAD graphical data resolution and direct weather viewing on pilots' judgments of weather severity and their willingness to continue a flight," (Technical Report no. DOT/FAA/AM-04/5). Washington, DC: Federal Aviation Administration, 2004.

[7] M.A. Burgess, and R.P. Thomas, "The effect of NEXRAD image looping and National Convective Weather Forecast Product on pilot decision making in the use of a cockpit weather information display," (Report no. NASA/CR-2004-212655). Hampton, VA: NASA Langley Research Center, 2004.
[8] W.R. Knecht, and E. Frazier, "The influence of looping NextGeneration Radar on general aviation pilots' flight Into adverse weather," (Technical Report no. DOT/FAA/AM-15/16). Washington, DC: Federal Aviation Administration, 2015.

[9] L. Hua, L. "Effect of age of radar information on the pilot's weather-related judgment," Unpublished doctoral dissertation, University of Oklahoma, Norman, 2014.

[10] K. Lemos, and J. Chamberlain, "In-flight weather trending information: Optimal looping characteristics for animated NEXRAD images," (Article no. 5.D.4). Proceedings of 23rd Digital Avionics Systems Conference, 2004.

[11] P.F. Novacek, M.A. Burgess, M.L. Heck, and A.F. Stokes, "The effect of ownship information and NexRad resolution on pilot decision making in the use of a cockpit weather information display," (Report No. NASA/CR-2001-210845). Hampton, VA: NASA Langley Research Center, 2001.

[12] S-C. Wu, C.G. Duong, R.W. Koteskey, and W.W. Johnson, "Designing a flight deck predictive weather forecast interface supporting trajectory-based operations," Ninth USA/Europe Air Traffic Management Research and Development Seminar, 2011.

[13] S-C Wu, C.L Gooding, A.E. Shelley, C.G. Duong, and W.W. Johnson, "Pilot convective weather decision making in en route airspace," 28th International Congress of the Aeronautical Sciences, 2012.

[14] D.E. Yuchnovicz, P.F. Novacek, M.A. Burgess, M.L. Heck, and A.F. Stokes, "Use of a data-linked weather information dis-play and effects on pilot decision making in a piloted simulation study," (Report no. NASA CR 2001-211047). Langley, VA: NASA Langley Research Center, 2001.

[15] Federal Aviation Administration, "Advisory Circular 00-24C," Feb. 19, 2013.

[16] G. Westheimer, "Visual acuity and hyperacuity," Investigative Ophthalmology, 14(8), pp. 570-572, 1975.

[17] J.J. Gibson, "The ecological approach to visual perception," New York: Houghton-Mifflin, 1979.

[18] D. Marr, "Vision: A computational investigation into the human representation and processing of visual information," San Francisco: W.H. Freeman, 1982

[19] "Mathematica," Wolfram Research, 2015.

[20] Wolfram Research, "Numerical nonlinear global optimization," in Constrained Optimization, 2013.

[21] Federal Aviation Administration, "Advisory Circular 00-24C," Feb. 19, 2013.

[22] National Transportation Safety Board, NTSB Safety Alert SA 017. Washington, DC: NTSB, 2012. 